\title{
RIBBON CONCORDANCE DOES NOT IMPLY A DEGREE ONE MAP
}

\author{
KATURA MIYAZAKI \\ (Communicated by Frederick R. Cohen)
}

\begin{abstract}
We give an example of classical knots $K_{0}, K_{1}$ such that (1) $K_{1}$ is ribbon concordant to $K_{0},(2)$ there are no degree one maps from the exterior of $K_{1}$ in $S^{3}$ to that of $K_{0}$.
\end{abstract}

1.

Throughout this note let $K_{0}$ and $K_{1}$ denote classical knots, $A_{i}$ denote the Alexander module of $K_{i}$, and $X_{i}$ the exterior of $K_{i}$ in $S^{3}$ for $i=0,1$. Let $\Lambda=Z\left[t, t^{-1}\right]$.

In [2] Gordon introduced the notion of ribbon concordance. We say $K_{1}$ is ribbon concordant to $K_{0}$ (and write $K_{1} \geq K_{0}$ ) if there is an annulus $C$ in $S^{3} \times I$ such that $C \cap S^{3} \times\{i\}=K_{i}, i=0,1$, and the restriction to $C$ of the projection $S^{3} \times I \rightarrow I$ is a Morse function with no local maxima. Gordon asked:

Question $1([2], 6.4)$. Let $v\left(K_{i}\right)$ denote the Gromov norm of $X_{i}$. Does $K_{1} \geq K_{0}$ imply $v\left(K_{1}\right) \geq v\left(K_{0}\right)$ ?

If there were a degree one map from $X_{1}$ to $X_{0}$, then an affirmative answer to the question would follow from the property of the Gromov norm. Such a degree one map would also imply that $A_{0}$ be a quotient of $A_{1}$. This is observed by Gilmer [1], and he asks:

Question 2 ([1], 4.6). Does $K_{1} \geq K_{0}$ imply that there is a $\Lambda$-epimorphism from $A_{1}$ to $A_{0}$ ?

In this paper we give a negative answer to this question.

Proposition 1. There are $K_{0}$ and $K_{1}$ such that

(1) $K_{1} \geq K_{0}$,

(2) there are no $\Lambda$-epimorphisms from $A_{1}$ to $A_{0}$.

In particular, there are no degree one maps from $X_{1}$ to $X_{0}$.

Received by the editors January 4, 1989.

1980 Mathematics Subject Classification (1985 Revision). Primary 57M25; Secondary 57Q60.

Key words and phrases. Ribbon concordance, degree one map, the ring of integers, Dedekind domain. 
In fact Gilmer's question is posed in an algebraically generalized form, but the proposition still gives a "no" answer. However, Question 1 remains open.

I would like to thank Professor Gordon for suggesting this problem to me.

2.

Let $K_{0}$ be a knot with a Seifert matrix $V_{0}$ below. Let $K_{1}$

$$
V_{0}=\left(\begin{array}{cc}
13 & 1 \\
0 & 1
\end{array}\right) \quad V_{1}=\left(\begin{array}{cccc}
13 & 1 & 0 & 3 \\
0 & 1 & 0 & 0 \\
0 & 0 & 0 & 2 \\
3 & 0 & 1 & 1
\end{array}\right)
$$

be a knot with a Seifert matrix $V_{1}$ and $K_{1} \geq K_{0}$. The existence of $K_{1}$ is guaranteed by [1, Theorem (1.3)]. Simplify the presentation matrix $t V_{1}-V_{1}^{T}$ of $A_{1}$ as follows.

$$
\begin{aligned}
t V_{1}-V_{1}^{T} & =\left(\begin{array}{cccc}
13(t-1) & t & 0 & 3(t-1) \\
-1 & t-1 & 0 & 0 \\
0 & 0 & 0 & 2 t-1 \\
3(t-1) & 0 & t-2 & t-1
\end{array}\right) \\
& \rightarrow\left(\begin{array}{cccc}
0 & 13(t-1)^{2}+t & 0 & 3(t-1) \\
-1 & t-1 & 0 & 0 \\
0 & 0 & 0 & 2 t-1 \\
3(t-1) & 0 & t-2 & t-1
\end{array}\right) \\
& \rightarrow\left(\begin{array}{cccc}
0 & 13(t-1)^{2}+t & 0 & * \\
-1 & 0 & 0 & 0 \\
0 & 0 & 0 & 2 t-1 \\
3(t-1) & 3(t-1)^{2} & t-2 & * *
\end{array}\right) \\
& \rightarrow\left(\begin{array}{ccc}
13 t^{2}-25 t+13 & 0 & * \\
0 & 0 & 2 t-1 \\
3(t-1)^{2} & t-2 & * *
\end{array}\right) \\
& \rightarrow\left(\begin{array}{ccc}
13 t^{2}-25 t+13 & 0 & * \\
0 & 0 & 2 t-1 \\
3 & t-2 & * *
\end{array}\right) \equiv M .
\end{aligned}
$$

Therefore $A_{1}$ is generated by three elements, say $\alpha, \beta$ and $\gamma$, subject to the relations

$$
M\left(\begin{array}{l}
\alpha \\
\beta \\
\gamma
\end{array}\right)=0 .
$$

It is easy to see that $A_{0}$ is the cyclic $\Lambda$-module of order $13 t^{2}-25 t+13$. Note that $A_{0}$ contains $(t-1)^{-1}$. 
Lemma 1. Suppose that there is a $\Lambda$-epimorphism $f: A_{1} \rightarrow A_{0}$. Then there are $u$ and $v$ in $A_{0}$ such that

$$
3 u+(x-1) v=0 \quad \text { and }(u, v)=(1), \quad \text { where } x=(t-1)^{-1} .
$$

Proof. Then $f(\alpha), f(\beta)$ and $f(\gamma)$ generate $A_{0}$ and satisfy the following equations.

$$
\begin{gathered}
\left(13 t^{2}-25 t+13\right) f(\alpha)+* f(\gamma)=0, \\
(2 t-1) f(\gamma)=0, \\
3 f(\alpha)+(t-2) f(\beta)+* * f(\gamma)=0 .
\end{gathered}
$$

Since $13 t^{2}-25 t+13$ is irreducible in $\Lambda,(2)$ shows $f(\gamma)=0$. It follows from (3) that

$$
3 f(\alpha)+(t-2) f(\beta)=0 .
$$

Multiplying by $x=(t-1)^{-1}$, we get

$$
3 x f(\alpha)+(1-x) f(\beta)=0 .
$$

The desired equation follows by setting $u=x f(\alpha)$ and $v=-f(\beta)$.

We shall show that $A_{0}$ does not have the elements $u$ and $v$ as in Lemma 1 . Express $A_{0}$ in terms of $x$ where $x=(t-1)^{-1}$; then $A_{0}$ is the cyclic $Z[1+$ $\left.x^{-1},\left(1+x^{-1}\right)^{-1}\right]$ module of order $1+x^{-1}+13 x^{-2}$. Since $x \in A_{0}$ and $\left(1+x^{-1}\right)^{-1}=x(x+1)^{-1}$. We obtain:

$$
A_{0} \cong Z\left[x, x^{-1},(1+x)^{-1}\right] /\left(x^{2}+x+13\right) \text {. }
$$

Let $D=Z[x] /\left(x^{2}+x+13\right)$ and $S$ be the multiplicative set of $D$ generated by $x$ and $x+1$. It follows that $A_{0} \cong S^{-1} D$. Since $D$ is $Z[(-1+\sqrt{-51}) / 2]$ which is the ring of integers in $Q(\sqrt{-51})$, in particular a Dedekind domain. In fact $A_{0}$ is also a Dedekind domain. The following algebraic lemmas establish Proposition 1.

Lemma 2. If an ideal $P$ is prime and nonprincipal in $D$, then so is $P S^{-1} D$ in $S^{-1} D$.

Lemma 3. In $D$ the following hold:

(1) $(3)=(3, x-1)^{2}$,

(2) $(x-1)=(3, x-1)(5, x-1)$,

(3) $(3, x-1)$ and $(5, x-1)$ are prime but nonprincipal ideals in $D$, (and hence also in $S^{-1} D$ by Lemma 2).

Proof of Proposition 1. If there is an epimorphism from $A_{1}$ to $A_{0}$, by Lemma 1 there are $u$ and $v$ in $S^{-1} D$ such that $3 u+(x-1) v=0$ and $(u, v)=(1)$. We have the ideal equation

$$
(3)(u)=(x-1)(v) \text {. }
$$


By Lemma 3 we obtain

$$
(3, x-1)^{2}(u)=(3, x-1)(5, x-1)(v) \text { in } S^{-1} D .
$$

Since an ideal in the Dedekind domain $S^{-1} D$ has a unique prime ideal decomposition, it follows that:

$$
(u)=(5, x-1) Q \text { and }(v)=(3, x-1) Q \text { for some ideal } Q .
$$

The ideal $(u)$ is principal, but $(5, x-1)$ is not. It follows that $Q \neq(1)$. Thus $(u, v)=Q \neq(1)$, a contradiction to Lemma 1 . The proof is completed.

Proof of Lemma 2. If $P \cap S \neq \varnothing, P$ contains a prime element $x$ or $x+1$. Thus $P$ is a principal ideal, a contradiction. It follows $P \cap S=\varnothing$. Then $P S^{-1} D$ is prime. If $P S^{-1} D$ is principal, there is a $b \in P$ such that $P S^{-1} D=b S^{-1} D$. Among all such $b$ take one such that $b D$ is maximal. This is possible because $D$ is Noetherian. Then $b$ is not divisible by $x$ or $x+1$. For an arbitrary $p \in P$ there are $s_{1}, s_{2} \in S$ and $d \in D$ such that $p / s_{1}=b d / s_{2}$. Thus $s_{2} p=b y$ for some $y \in D$. Let $s_{2}=p_{1} \cdots p_{r}$ with $p_{i}=x$ or $x+1$; then $p_{i} \nmid b$, so that $p_{i} \mid y$. An induction on $r$ shows that $y$ is divisible by $s_{2}$, so $p \in b D$. It follows that $P=b D$. This contradicts the assumption that $P$ is not principal. Thus $P S^{-1} D$ is not principal.

Proof of Lemma 3. Since $D /(3, x-1)=Z_{3}[x] /(x-1) \cong Z_{3}$, a domain, (3, $x-1)$ is a prime ideal of norm 3 . On the other hand we see that $(3, x-1)^{2} \subset$ (3), for $(x-1)^{2}=-3(x+4)$ in $D$. Since the norm of $(3, x-1)^{2}$ is 9 , it follows $(3, x-1)^{2}=(3)$.

If $(3, x-1)$ were principal, it could be written as

$$
\left(a+b \frac{-1+\sqrt{-51}}{2}\right) \quad \text { where } a, b \in Z \text {. }
$$

Then the norm of $(3, x-1)$ is $\left((2 a-b)^{2}+51 b^{2}\right) / 4$, which must be 3 . However, there are no integral solutions of

$$
(2 a-b)^{2}+51 b^{2}=12 .
$$

Thus $(3, x-1)$ is not principal. By the similar arguments we can prove the conclusions about $(x-1)$ and $(5, x-1)$.

\section{REFERENCES}

1. P. Gilmer, Ribbon concordance and a partial order on S-equivalence classes, Topology Appl. 18 (1984), 121-144.

2. C. Gordon, Ribbon concordance of knots in the 3-sphere, Math. Ann. 257 (1981), 157-170.

Department of Mathematics, University of Texas at Austin, Austin, Texas 78712 